\title{
COVID-19 diagnostic testing and the psychology of precautions fatigue
}

\begin{abstract}
65-YEAR-OLD MAN is hospitalized with mild A dyspnea and myalgias. After an initial nasopharyngeal swab is negative for SARS-CoV-2, the hospital staff requests that special respiratory precautions be discontinued without a second test. They cite a low observed yield of repeated testing and the burden of precautions on workflow.

Hospital policies regarding the necessity of a confirmatory second negative test are based on the chances of an initial false negative in a population with an estimated probability of disease. However, staff perception of residual disease risk is based on the chances of a patient's having COVID-19 despite having tested negative. Psychological fatigue arises from incessant gowning, gloving, and masking among patients extremely unlikely to have COVID-19. This fatigue leads to a reduced vigilance that degrades the value of precautions across all patients in isolation.
\end{abstract}

\section{PRECAUTIONS FATIGUE IS ANALOGOUS TO ALARM FATIGUE}

The COVID-19 crisis is an opportunity to reframe clinical decision-making. Despite decades of teaching clinical reasoning, promulgating practice guidelines, and advocating shared decision-making to allocate scarce resources, excessive testing and monitoring persist. One consequence is alarm fatigue, ${ }^{1}$ caused by false-positive (clinically insignificant) notifications of status changes-when alarms persistently sound with little purpose, people stop paying attention.

A generation ago, analogous concerns generated surgeons' desire for human immunodeficiency virus screening to enable selec-

doi:10.3949/ccjm.88a.20086 tive implementation of special precautions rather than universally applied intensive measures. The large number of COVID-19 patients and hospitalized "patients under investigation" (PUIs) for whom special respiratory precautions are ordered is creating a previously unseen degree of "precautions fatigue." The current practice environment represents an opportunity for experiential cognizance of well-intentioned efforts at error avoidance that become diluted through overly broad application.

With the increased incidence of disease in the community, more readily available testing, recognition of nosocomial transmission, and fewer admissions for diseases other than COVID-19, the proportion of hospitalized patients being evaluated for COVID-19 is markedly higher than it was earlier in the pandemic. When testing was scarce, costly, and time-consuming, patients were tested only if they had multiple COVID-19 symptoms and a known exposure or relevant travel history. With increasing incidence, populations being tested have broadened, escalating the number of patients considered at risk and creating anxiety among healthcare workers through both the presence of true disease and the ubiquitous respiratory precautions signs on isolation rooms. ${ }^{2}$

\section{CONSEQUENCES \\ OF PRECAUTIONS FATIGUE}

Unless incremental increases in testing are specifically targeted at high-prevalence groups, higher testing rates generally result in both a lower proportion of positive test results (lower diagnostic yield due to reduced average pretest probability) and less-severe disease, on average, among those who test positive. ${ }^{3}$ Since hospital-
The COVID-19

crisis is an opportunity to reframe clinical decisionmaking 
ized PUIs affect behavior of hospital staff and deplete personal protective equipment, it is important to understand their effect on the psychology of inpatient healthcare workers and on disease transmission.

Precautions fatigue has become evident in hospitals, reducing the average effectiveness of precautions the same way that alarm fatigue desensitizes staff, reducing the average clinical benefit of each alarm triggered. ${ }^{1}$ Precautions fatigue results in guideline-discordant reuse of personal protective equipment, reduced attention to facial protection (given barriers to its access), less-vigorous encouragement of patient mask-donning, and dramatic reductions in direct patient contact.

Early in the pandemic, a substantial proportion of hospitalized PUIs were eventually diagnosed with COVID-19, for several reasons. Testing had a relatively high yield because protocols restricted testing to high-risk populations, as understanding of the clinical spectrum of disease was poor and testing capability and availability were limited. Testing had higher sensitivity due to policies (inadvertently) restricting testing to patients with greater viral shedding and substantial nasal secretions. ${ }^{4}$ Also, there was a time lag between sending the sample and getting the result, so that for PUIs, initial results of nasopharyngeal swabs were not available for 2 to 3 days, leaving them at a higher instantaneous probability of disease, ie, probably at one point in time.

More recently, when the clinical presentation suggests the disease, most hospitalized PUIs have received negative results on their first test before being admitted, which reduces the probability of disease to the false-negative rate. The exceptions are those with a positive first test (who would be admitted to a COVID-19 unit and would no longer be considered under investigation) and those with a pretest probability sufficiently low for liberation from PUI status after a single negative test.

\section{FALSE-NEGATIVE RATES VS FALSE-OMISSION RATES}

Diagnostic tests have known or imperfectly estimated sensitivities and specificities, which depend on the tests themselves and factors such as sample quality, body habitus, and tim- ing of sampling relative to the natural history of disease. Although the sensitivity of polymerase chain reaction testing of a nasopharyngeal swab for SARS-CoV-2 has not been established, many hospitals have been using an estimate of $70 \%$, based on early studies. ${ }^{5}$

As a test characteristic, sensitivity does not generally depend on disease prevalence in the population tested. However, if less-symptomatic populations and asymptomatic preprocedure inpatients are tested, lower levels of viral shedding and lower volume of nasal secretions among those tested may decrease the sensitivity, as studies of influenza have demonstrated. ${ }^{4}$ Therefore, while we assume the sensitivity is $70 \%$, a lower value would increase both the false-negative rate and the false-omission rate (ie, 1 minus the negative predictive value).

A relatively low positive predictive value and high false-discovery rate (1 minus the positive predictive value) have been implicated in the poor response times associated with alarm fatigue. ${ }^{1}$ However, reductions over time in attentiveness to precautions among staff caring for COVID-19 PUIs is due to the aggregate of both true and perceived lower prevalence of disease, considering all those tested as the denominator. The true prevalence of disease is decreased due to increased testing rates and the fact that most PUIs have had a negative test result. The latter leads to a probability of disease given as:

\section{$P$ (negative test | COVID +)}

ie, the probability that a diseased patient would test negative, which is equal to the false-negative rate times the true prevalence within the tested population. However, staff perceptions of residual disease prevalence will be based on the false-omission rate, given as:

$P($ COVID + | negative test)

ie, the probability that a patient who tests negative has COVID, rather than the falsenegative rate.

Two relevant considerations arise from the distinction between precautions policies among those with a first negative test, which are often based on the false-negative rate. First, the false-omission rate varies much more with prevalence than the false-negative rate. For example, assuming the sensitivity is $70 \%$ and the prevalence is $10 \%$, the false-negative rate and false-omission rate are nearly identi- 


\section{RUHNKE}

cal at 0.03 and 0.0326 , respectively. However, if the prevalence is $50 \%$, the false-negative rate and false-omission rate diverge to 0.15 and 0.233 , respectively, creating clinically significant relative and absolute differences.

Moreover, let us assume the specificity is 99\%. Based on Fagan's nomogram and a calculated negative likelihood ratio of 0.30 , the posttest probability of disease at prevalence rates of $10 \%$ and $50 \%$ would be 0.03 and 0.23 , nearly indistinguishable from the false-omission rates.

Second, precautions-laden inpatient units reduce the perceived COVID-19 prevalence among isolation precautions hospital rooms, caused by true diminishing marginal returns to incremental increases in testing that occur as a result of surveillance bias. There is a substantial literature on the psychology of false positives ${ }^{6}$ but little empirical evidence regarding cognitive processes surrounding false negatives. Despite this, previous literature shows that everyday human cognitive judgments follow the statistical principles of perception and reveal a close correspondence between implicit human probabilistic models and empiric statistical models. ${ }^{7}$ Cognitive heuristics are operationalized based on predictive values and their complements, such that, in the same way that false-discovery rates drive alarm fatigue, false-omission rates are likely the principal psychological driver of precautions fatigue. The commonality of empiric statistics and psychologic processes is a rationale for using decrements in the false-omission rate as a quantitative measure of the contribution of incremental testing to diagnostic yield.

\section{RETURN TO THE CASE SCENARIO}

Since false-omission rates are more dependent on prevalence than false-negative rates, they should be considered in COVID-19 liberation pathways. Practically, this means that, in addition to the well-recognized adverse effects of precautions, ${ }^{8}$ decisions about liberating patients from COVID-19 precautions should consider the reduced clinical benefit of isolation if precautions fatigue causes noncompliance as an unintended consequence. Medical literature suggests that contact precautions are used more often than evidence warrants, which creates more harm than benefit. ${ }^{8}$ A learning healthcare system should use the COVID-19 experience to incorporate the real impact of precautions fatigue into decision algorithms and clinical pathways.

Protection of hospital staff and limiting nosocomial transmission of COVID-19 are critical. However, overuse of special precautions in patients unlikely to have true disease can lead to precautions fatigue that diminishes vigilance among staff, thereby vitiating the value of isolation. Hospital leadership should assertively select patients for testing and retesting, considering that precautions fatigue markedly reduces the average effectiveness of precautions for all isolation rooms.

\section{DISCLOSURES}

The author reports no relevant financial relationships which, in the context of his contribution, could be perceived as a potential conflict of interest.

\section{REFERENCES}

1. Winters BD, Cvach MM, Bonafide CP, et al. Technological distractions (part 2): a summary of approaches to manage clinical alarms with intent to reduce alarm fatigue. Crit Care Med 2018; 46(1):130137. doi:10.1097/CCM.0000000000002803

2. Shanafelt T, Ripp J, Trockel M. Understanding and addressing sources of anxiety among health care professionals during the COVID-19 pandemic. JAMA 2020; 323(21):2133-2134. doi:10.1001/jama.2020.5893

3. Verity R, Okell LC, Dorigatti I, et al. Estimates of the severity of coronavirus disease 2019: a model-based analysis. Lancet Infect Dis 2020; 20(6):669-677. doi:10.1016/S1473-3099(20)30243-7.

4. Chartrand C, Leeflang MM, Minion J, Brewer T, Pai M. Accuracy of rapid influenza diagnostic tests: a meta-analysis. Ann Intern Med 2012; 156(7):500-511.

doi:10.7326/0003-4819-156-7-201204030-00403
5. Zitek T. The appropriate use of testing for COVID-19. West J Emerg Med 2020; 21(3):470-472. doi:10.5811/westjem.2020.4.47370

6. Simmons JP, Nelson LD, Simonsohn U. False-positive psychology: undisclosed flexibility in data collection and analysis allows presenting anything as significant. Psychol Sci 2011; 22(11):1359-1366. doi:10.1177/0956797611417632

7. Griffiths TL, Tenenbaum JB. Optimal predictions in everyday cognition. Psychol Sci 2006; 17(9):767-773. doi:10.1111/j.1467-9280.2006.01780.x

8. Young K, Doernberg SB, Snedecor RF, Mallin E. Things we do for no reason: contact precautions for MRSA and VRE. J Hosp Med 2019; 14(3):178-180. doi:10.12788/jhm.3126

Address: Gregory W. Ruhnke, MD, MS, MPH, Section of Hospital Medicine, Department of Medicine, University of Chicago, 5841 South Maryland Avenue, MC 5000, Chicago, IL 60637;

gruhnke@medicine.bsd.uchicago.edu 